\title{
Mixed QCD-EW corrections to Drell-Yan at the LHC
}

\author{
Luca Buonocore ${ }^{a, *}$ \\ ${ }^{a}$ Universität Zürich, Physik Institut \\ Winterthurerstrasse 190, CH-8057 Zürich, Switzerland \\ E-mail: 1buono@physik.uzh.ch
}

We discuss recent theoretical results for the complete mixed QCD-EW corrections to leptonspair production via the Drell-Yan mechanism, a cornerstone process for the precision physics programme at the LHC. We present results for fiducial cross sections and differential distributions to both the neutral current- and charged current- process. We show that the mixed corrections are relatively large and of similar size to the NNLO QCD ones at central scales. In particular, for the neutral current case, we report the first result at this order that is valid in the entire range of dilepton invariant masses.

*** The European Physical Society Conference on High Energy Physics (EPS-HEP2021), *** *** 26-30 July $2021 * * *$

*** Online conference, jointly organized by Universität Hamburg and the research center DESY ***

\footnotetext{
${ }^{*}$ Speaker
} 


\section{Introduction}

In view of the absence of striking signals for beyond the Standard Model phenomena at the CERN Large Hadron Collider (LHC), alternative routes must be pursued to uncover possible new physics effects. A viable strategy is the search for small deviations from the predictions of the Standard Model and precision is the key for this path.

Lepton-pair production through an off-shell vector boson, namely the Drell-Yan mechanism, represents a cornerstone process for the precision physics programme at the LHC. It features large production rates and clean experimental signatures, given the presence of at least one lepton with large transverse momentum in the final state. Therefore, it is of primary importance for the extraction of crucial electro-weak parameters of the Standard Model, as the $W$ boson mass and the Weinberg angle, as well as input to the determination of parton distribution functions (PDFs). Furthermore, the Drell-Yan process is also very important in the context of new physics searches, severely constraining many possible scenarios.

The Drell-Yan process is one of the most studied and well known processes. Inclusive cross sections have been recently pushed to the $\mathrm{N}^{3} \mathrm{LO}$ level for the production of a virtual photon [1, 2] and of $W$ boson [3]. Since predictions for differential observables including leptonic decays are known up to NNLO in QCD and and up to NLO in EW, the attention of the theory community has recently turned to the mixed QCD-EW corrections.

In this contribution, we will present recent results for the mixed corrections to both neutral current- and charged current- Drell-Yan process, showing their impact on fiducial cross sections and on a selection of kinematical distributions.

\section{Neutral current Drell-Yan process}

We focus on the hadroproduction of a massive lepton-anti-lepton pair, namely the process

$$
p p \rightarrow \ell^{+} \ell^{-}+X
$$

and we introduce the following notation for the corresponding differential cross section

$$
d \sigma=\sum_{m, n=0}^{\infty} d \sigma^{(m, n)},
$$

where $d \sigma^{(0,0)} \equiv d \sigma_{\mathrm{LO}}$ is the Born level contribution and $d \sigma^{(m, n)}$ the $O\left(\alpha_{\mathrm{S}}^{m} \alpha^{n}\right)$ correction. In particular, the term $m=n=1$ corresponds to mixed QCD-EW corrections.

The computation of $O\left(\alpha_{\mathrm{S}} \alpha\right)$ mixed corrections to the full $2 \rightarrow 2$ Drell-Yan process Eq. (1) is a formidable task. The evaluation of the corresponding $2 \rightarrow 2$ two-loop amplitudes involves scalar integrals with internal masses at the frontier of current computational techniques. Furthermore, it presents a rich infra-red (IR) structure involving both initial- and final- state radiation. The cancellation of the corresponding IR singularities requires a suitable NNLO subtraction scheme.

Very recently the exact mixed corrections have been achieved in Ref. [4]. The two-loop virtual contribution has been worked out by using semi-analytical techniques, overcoming the technical problems in the evaluation of the relevant master integrals. The cancellation of the IR divergences 


\begin{tabular}{|c|c|c|c|c|c|}
\hline$\sigma[\mathrm{pb}]$ & $\sigma_{\mathrm{LO}}$ & $\sigma^{(1,0)}$ & $\sigma^{(0,1)}$ & $\sigma^{(2,0)}$ & $\sigma^{(1,1)}$ \\
\hline \hline$q \bar{q}$ & $809.56(1)$ & $191.85(1)$ & $-33.76(1)$ & $49.9(7)$ & $-4.8(3)$ \\
\hline$q g$ & - & $-158.08(2)$ & - & $-74.8(5)$ & $8.6(1)$ \\
\hline$q(g) \gamma$ & - & - & $-0.839(2)$ & - & $0.084(3)$ \\
\hline$q(\bar{q}) q^{\prime}$ & - & - & - & $6.3(1)$ & $0.19(0)$ \\
\hline$g g$ & - & - & - & $18.1(2)$ & - \\
\hline$\gamma \gamma$ & $1.42(0)$ & - & $-0.0117(4)$ & - & - \\
\hline \hline tot & $810.98(1)$ & $33.77(2)$ & $-34.61(1)$ & $-0.5(9)$ & $4.0(3)$ \\
\hline \hline$\sigma / \sigma_{\mathrm{LO}}$ & 1 & $4.2 \%$ & $-4.3 \%$ & $0 \%$ & $+0.5 \%$ \\
\hline
\end{tabular}

(a) $p p \rightarrow \mu^{+} \mu^{-}+X$.

\begin{tabular}{|c|c|c|c|c|c|}
\hline$\sigma[\mathrm{pb}]$ & $\sigma_{\mathrm{LO}}$ & $\sigma^{(1,0)}$ & $\sigma^{(0,1)}$ & $\sigma^{(2,0)}$ & $\sigma^{(1,1)}$ \\
\hline \hline$q \bar{q}$ & 5029.2 & $970.5(3)$ & $-143.61(15)$ & $251(4)$ & $-7.0(1.2)$ \\
\hline$q g$ & - & $-1079.86(12)$ & - & $-377(3)$ & $39.0(4)$ \\
\hline$q(g) \gamma$ & - & - & $2.823(1)$ & - & $0.055(5)$ \\
\hline$q(\bar{q}) q^{\prime}$ & - & - & - & $44.2(7)$ & $1.2382(3)$ \\
\hline$g g$ & - & - & - & $100.8(8)$ & - \\
\hline \hline tot & 5029.2 & $-109.4(4)$ & $-140.8(2)$ & $19(5)$ & $33.3(1.3)$ \\
\hline \hline$\sigma / \sigma_{\mathrm{LO}}$ & 1 & $-2.2 \%$ & $-2.8 \%$ & $+0.4 \%$ & $+0.6 \%$ \\
\hline
\end{tabular}

(b) $p p \rightarrow \mu^{+} v_{\mu}+X$.

Table 1: The different perturbative contributions to the fiducial cross section and their breakdown into the various partonic channels (for $\mu_{\mathrm{R}}=\mu_{\mathrm{F}}=m_{Z}$ ). The numerical uncertainties are stated in brackets.

has been achieved by exploiting the $q_{T}$ subtraction formalism [5]. The extension of the method to the NLO EW and the mixed QCD-EW corrections have been worked out in Refs. [6] and [7], starting from the $q_{T}$ subtraction formalism for heavy quarks [8].

\subsection{Results}

We show results for the process $p p \rightarrow \mu^{+} \mu^{-}+X$ at centre-of-mass energy $\sqrt{s}=14 \mathrm{TeV}$ with the setup of Ref. [4]. In particular, we use the following selection cuts,

$$
p_{T, \mu^{ \pm}}>25 \mathrm{GeV}, \quad\left|y_{\mu^{ \pm}}\right|<2.5, \quad m_{\mu \mu}>50 \mathrm{GeV} .
$$

We work at the level of bare muons, i.e., no lepton recombination with close-by photons is carried out. In Table 1a we present predictions for the corresponding fiducial cross section at central scales $\mu_{\mathrm{R}}=\mu_{\mathrm{F}}=m_{Z}$. We show the breakdown of the different contributions $\sigma^{(i, j)}$ into the various partonic channels. The contribution from quark-antiquark annihilation is denoted by $q \bar{q}$. The contributions from the channels $q g+\bar{q} g$ and $q \gamma+\bar{q} \gamma+g \gamma$ are labelled by $q g$ and $q(g) \gamma$, respectively. The contribution from all the remaining quark-quark channels $q q^{\prime}, \bar{q} \bar{q}^{\prime}$ (including both $q=q^{\prime}$ and $q \neq q^{\prime}$ ) and $q \bar{q}^{\prime}$ (with $q \neq q^{\prime}$ ) is labelled by $q(\bar{q}) q^{\prime}$. Finally, the contributions from the gluon-gluon and photon-photon channels are denoted by $g g$ and $\gamma \gamma$, respectively.

We see that the radiative corrections are subject to large cancellations between the various partonic channels. The NLO QCD corrections $\sigma^{(1,0)}$ and the NLO EW corrections $\sigma^{(0,1)}$ have a similar size and opposite sign, leading to an additional cancellation. The NNLO QCD corrections give an essentially vanishing contribution within the numerical uncertainties. The QCD-EW corrections are relatively large and amounts to $+0.5 \%$ with respect to the $\mathrm{LO}$ result.

In Fig. 1 we show on the left the $O\left(\alpha_{\mathrm{S}} \alpha\right)$ correction as a function of the anti-muon $p_{T}$. The results for the complete $O\left(\alpha_{\mathrm{S}} \alpha\right)$ correction are compared with those obtained in two approximations. The first approximation consists in computing the finite part of the two-loop virtual amplitude in the pole approximation [9]. Following the same strategy adopted for the charged-current Drell-Yan process in Ref. [7], for which the two-loop amplitude is not available yet, a suitable reweighting procedure with the exact squared Born amplitude is performed.

The second approximation is based on a fully factorised approach for QCD and EW corrections, where we exclude photon-induced processes throughout (see Ref. [7, 10] for a detailed description). 

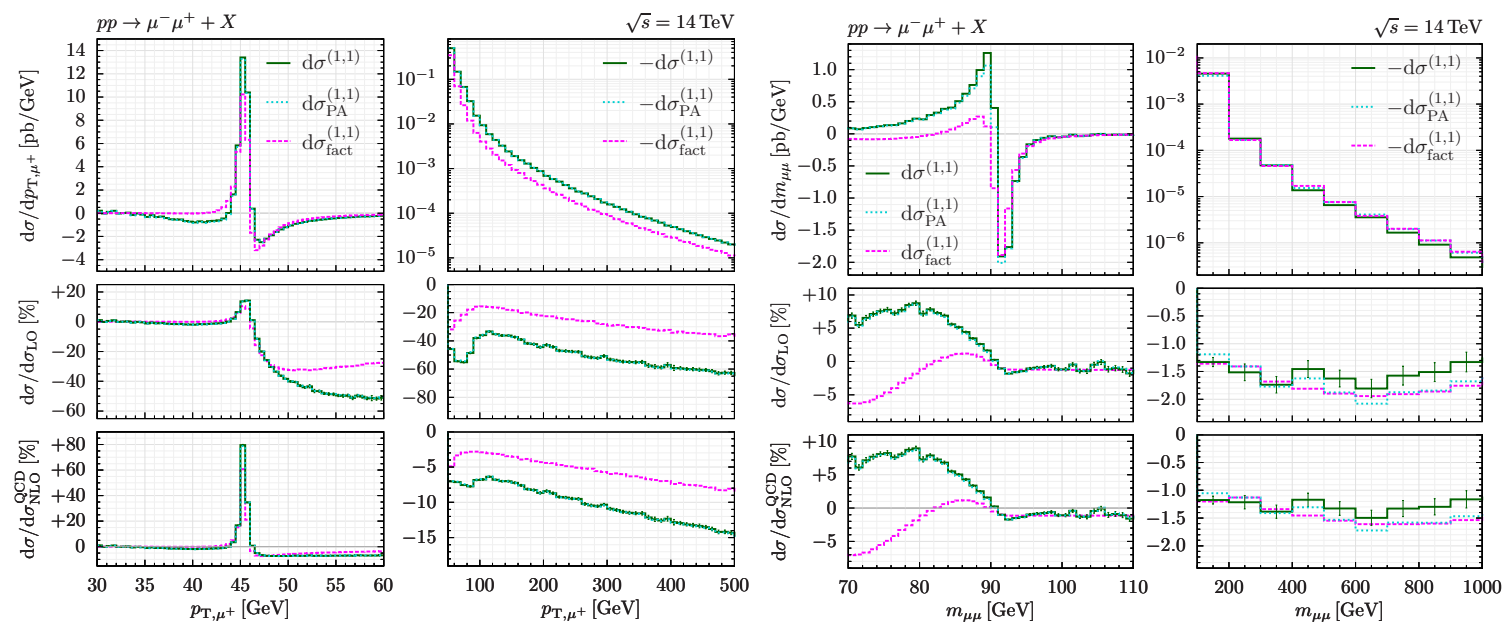

Figure 1: Complete $O\left(\alpha_{\mathrm{S}} \alpha\right)$ corrections to the differential cross section $d \sigma^{(1,1)}$ in the anti-muon $p_{T}$ (left) and muon-anti-muon invariant mass $m_{\mu^{+}} \mu^{-}$(right), compared to the corresponding result in the pole approximation and to the factorised approximation $d \sigma_{\text {fact }}^{(1,1)}$. The top panels show the absolute predictions, while the central (bottom) panels display the $O\left(\alpha_{\mathrm{S}} \alpha\right)$ correction normalized to the LO (NLO QCD) result. For the full result, the ratios also display the combination of statistical and systematic uncertainties associated to the subtraction method as explained in Ref. [4].

We see that the result obtained in the pole approximation is in perfect agreement with the exact result. This is due to the small contribution of the two-loop virtual to the computed correction.

As $p_{T}$ increases, the (negative) impact of the mixed QCD-EW corrections increases, and at $p_{T}=500 \mathrm{GeV}$ it reaches about $-60 \%$ with respect to the $\mathrm{LO}$ prediction and $-15 \%$ with respect to the NLO QCD result.

In Fig. 1, we show on the right the result for the $O\left(\alpha_{\mathrm{S}} \alpha\right)$ correction as a function of the di-muon invariant mass $m_{\mu \mu}$. We notice that the pure factorised result fails to describe the exact radiative correction below the $Z$ resonance. In contrast, the pole approximation is a very good approximation of the complete correction, with some small differences that can be appreciated right around the peak. In the high $-m_{\mu \mu}$ region the correction is uniformly of the order of $-1.5 \%$ with respect to the NLO QCD result. Here the trend of the negative correction is captured by both approximations, which, however, both undershoot the exact result by about $30 \%$, highlighting the relevance of the exact two-loop contribution for this observable.

\section{Charged current Drell-Yan process}

The mixed QCD-EW corrections to the charged current Drell-Yan process has been computed in Ref. [7]. All real and virtual contributions are consistently included but for the two-loop virtual amplitude. As already mentioned in the previous section, the latter is approximated by its expansion around the resonant pole, applying the Pole Approximation and improved via a reweighting prescription. The quality of this approximation has been discussed in Ref. [7] and strongly confirmed by the comparison with the exact result for the neutral current case (see previous section). 

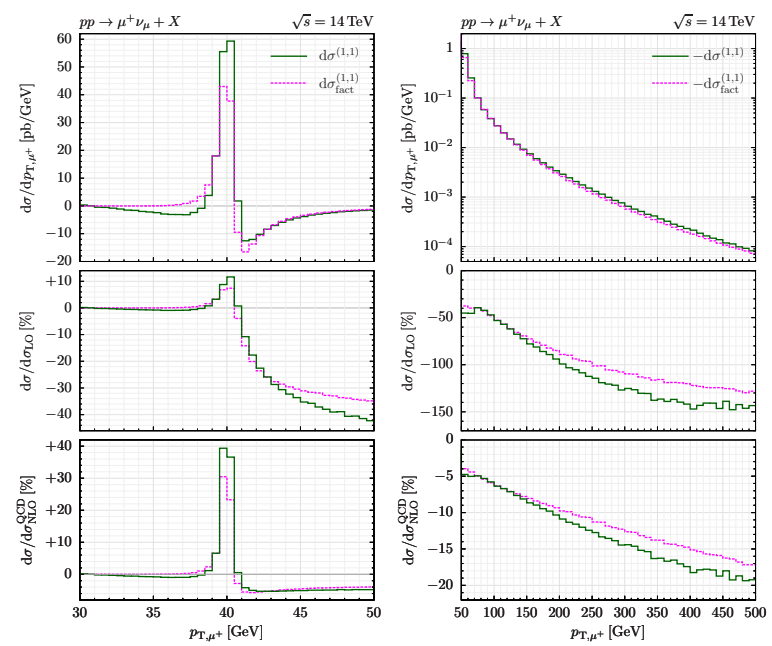

Figure 2: As is in Fig. 1 for the transverse momentum spectrum of the positively charged muon.

\subsection{Results}

We show results for the process $p p \rightarrow \mu^{+} v_{\mu}^{-}+X$ at centre-of-mass energy $\sqrt{s}=14 \mathrm{TeV}$ with the setup of Ref. [7]. In particular, we use the following selection cuts,

$$
p_{T, \mu}>25 \mathrm{GeV}, \quad\left|y_{\mu}\right|<2.5, \quad p_{T, v}>25 \mathrm{GeV},
$$

and work at the level of bare muons. In Table $1 \mathrm{~b}$ we present predictions for the corresponding fiducial cross section at central scales $\mu_{\mathrm{R}}=\mu_{\mathrm{F}}=m_{Z}$. We see large cancellations among the partonic channels, similarly to what observed for the neutral current case, see Table 1a. Also here, the mixed QCD-EW corrections are relatively large, being of similar size to the NNLO QCD ones. Because of the large cancellations occurring for central scales, we observe that the pattern of the higher-order QCD corrections to the perturbative series has a strong dependence on the choice of the renormalization and factorisation scales. For example, the scale choice $\mu_{R}=\mu_{F}=m_{W} / 2$ leads to a more common perturbative pattern: $\sigma^{(1,0)} / \sigma_{\mathrm{LO}}=+10 \%, \sigma^{(0,1)} / \sigma_{\mathrm{LO}}=-2.9 \%, \sigma^{(2,0)} / \sigma_{\mathrm{LO}}=$ $+4.2 \%, \sigma^{(1,1)} / \sigma_{\mathrm{LO}}=+0.76 \%$.

In Fig. 2, we show our results for the complete $O\left(\alpha_{\mathrm{S}} \alpha\right)$ correction to the transverse momentum spectrum of the positively charged muon. Similarly to the neutral current case, we compare our results with those obtained with a multiplicative combination of the NLO QCD and NLO EW corrections. We observe that overall the factorised approximation reproduces qualitatively well the complete results. As $p_{T}$ increases, the negative impact of the mixed QCD-EW corrections increases and becomes rather sizeable, reaching at $p_{T}=500 \mathrm{GeV}$ about $-140 \%$ with respect to the LO prediction and $-20 \%$ with respect to the NLO QCD result. This is not unexpected, since the high- $p_{T}$ region is dominated by $W+$ jet topologies, for which the $O\left(\alpha_{\mathrm{S}} \alpha\right)$ effects can be seen as NLO EW corrections.

\section{Conclusions}

We have presented results for mixed QCD-EW corrections to both the neutral current- and the charged current- Drell-Yan process. We have shown their impact on fiducial cross sections and 
selected differential observables, finding relatively large effects. In particular, for the neutral current case, we have shown the first result at this order that is valid in the entire range of dilepton invariant masses.

\section{Acknowledgments}

This material is based upon work supported in part by the Swiss National Science Foundation under contracts IZSAZ2_173357 and 200020_188464, by the ERC Starting Grant 714788 REINVENT, by the INFN, by the Italian Ministero dell'Università e della Ricerca Grant No. PRIN2017, by the European Research Council under the European Unions Horizon 2020 research and innovation Programme Grant Agreement No. 740006 and by the COST Action CA16201 PARTICLEFACE.

\section{References}

[1] C. Duhr, F. Dulat and B. Mistlberger, Drell-Yan Cross Section to Third Order in the Strong Coupling Constant, Phys. Rev. Lett. 125 (2020) 172001, [2001.07717].

[2] X. Chen, T. Gehrmann, N. Glover, A. Huss, T.-Z. Yang and H. X. Zhu, Di-lepton Rapidity Distribution in Drell-Yan Production to Third Order in QCD, 2107.09085.

[3] C. Duhr, F. Dulat and B. Mistlberger, Charged current Drell-Yan production at $N^{3} L O$, JHEP 11 (2020) 143, [2007.13313].

[4] R. Bonciani, L. Buonocore, M. Grazzini, S. Kallweit, N. Rana, F. Tramontano and A. Vicini, Mixed strong-electroweak corrections to the Drell-Yan process, 2106.11953.

[5] S. Catani and M. Grazzini, An NNLO subtraction formalism in hadron collisions and its application to Higgs boson production at the LHC, Phys. Rev. Lett. 98 (2007) 222002, [hep-ph/0703012].

[6] L. Buonocore, M. Grazzini and F. Tramontano, The $q_{T}$ subtraction method: electroweak corrections and power suppressed contributions, Eur. Phys. J. C 80 (2020) 254, [1911.10166].

[7] L. Buonocore, M. Grazzini, S. Kallweit, C. Savoini and F. Tramontano, Mixed QCD-EW corrections to $\boldsymbol{p} \boldsymbol{p} \rightarrow \boldsymbol{\ell} \boldsymbol{v}_{\boldsymbol{\ell}}+\boldsymbol{X}$ at the LHC, Phys. Rev. D 103 (2021) 114012, [2102.12539].

[8] S. Catani, S. Devoto, M. Grazzini, S. Kallweit, J. Mazzitelli and H. Sargsyan, Top-quark pair hadroproduction at next-to-next-to-leading order in QCD, Phys. Rev. D 99 (2019) 051501, [1901.04005].

[9] S. Dittmaier, A. Huss and C. Schwinn, Mixed $Q C D$-electroweak $O\left(\alpha_{s} \alpha\right)$ corrections to Drell-Yan processes in the resonance region: pole approximation and non-factorizable corrections, Nucl. Phys. B 885 (2014) 318-372, [1403 . 3216].

[10] S. Dittmaier, A. Huss and C. Schwinn, Dominant mixed QCD-electroweak $O\left(\alpha_{s} \alpha\right)$ corrections to Drell-Yan processes in the resonance region, Nucl. Phys. B 904 (2016) 216-252, [1511.08016]. 